\title{
Instructive signals for motor learning from visual cortical area MT
}

\author{
Megan R Carey ${ }^{1,2}$, Javier F Medina ${ }^{1}$, and Stephen G Lisberger ${ }^{1}$ \\ 1 Howard Hughes Medical Institute, W.M. Keck Foundation Center for Integrative Neuroscience, \\ Neuroscience Graduate Program, and Department of Physiology, University of California, San Francisco, \\ California 94143-0444, USA.
}

\section{Abstract}

Sensory error signals have long been proposed to act as instructive signals to guide motor learning. Here we have exploited the temporal specificity of learning in smooth pursuit eye movements and the well-defined anatomical structure of the neural circuit for pursuit to identify a part of sensory cortex that provides instructive signals for motor learning in monkeys. We show that electrical microstimulation in the motion-sensitive middle temporal area (MT) of extrastriate visual cortex instructs learning in smooth eye movements in a way that closely mimics the learning instructed by real visual motion. We conclude that MT provides instructive signals for motor learning in smooth pursuit eye movements under natural conditions, suggesting a similar role for sensory cortices in many kinds of learned behaviors.

We learn from our mistakes. Incorrect movements generally are followed by sensory feedback indicating that an error has been made. Many learning theories ${ }^{1-3}$ propose that neural representations of sensory error signals are the teachers for motor learning. Yet we understand only poorly how and where sensory error signals are converted into neural instructive signals that induce behavioral learning.

We have used smooth pursuit eye movements of rhesus monkeys to study the neural mechanisms of motor learning. Pursuit allows primates to track smoothly moving targets by matching eye velocity to target velocity 4,5 , thereby minimizing image motion relative to the eye. If a target changes direction or speed unexpectedly, then the delay in visual-motor processing for pursuit will result in image motion because there is a delay of $100 \mathrm{~ms}$ or more before eye velocity reacts to the new target velocity. If the same change in the direction or speed of target motion occurs repeatedly at a consistent time, then the visual to motor transformation for pursuit undergoes experience-dependent learning. Over time, the pursuit response to the initial target motion is altered so that it anticipates the change in target motion $^{6-8}$. Thus, pursuit shows associative learning that can be instructed by a consistent change in target trajectory. In real life, continual pursuit learning ensures excellent tracking of naturally-occurring object motion.

In the present paper, we have asked whether representations of image motion in area MT provide instructive signals for pursuit learning. MT neurons are selective for direction and speed of retinal image motion as well as a variety of other features of the visual stimulus 9 , 10. Prior studies have shown that visual motion signals for pursuit are represented in $\mathrm{MT}^{11-}$ 13 and that microstimulation of clusters of MT neurons with shared direction preferences has a directional effect on pursuit eye movements ${ }^{14,15}$ and perceptual judgments 16,17 . Here, we move beyond these previous observations to show that microstimulation creates instructive

Correspondence should be addressed to M.R.C. (megan_carey@hms.harvard.edu)..

${ }_{2}^{2}$ Present address: Department of Neurobiology, Harvard Medical School, 220 Longwood Avenue, Boston, Massachusetts 02115, USA.

COMPETING INTERESTS STATEMENT The authors declare that they have no competing financial interests. 
signals capable of driving learning in pursuit. Further, we demonstrate that the learning evoked by electrical stimulation in MT closely resembles that evoked by real visual motion, suggesting that activation of MT provides a powerful instructive signal for pursuit learning under natural conditions.

\section{RESULTS}

\section{Learning induced by microstimulation in MT}

We asked whether microstimulation in area MT, when consistently associated with motion of a pursuit target, would be sufficient to instruct directional learning of pursuit eye movements. Our procedure was designed to mimic experiments that used a precisely timed change in the direction of target motion as the instructive signal for directional learning in pursuit ${ }^{7}$, but here we replaced the change in target direction with MT microstimulation.

The basic pursuit stimulus consisted of individual trials of step-ramp target motion ${ }^{4}$. The target moved at $20^{\circ} \mathrm{s}^{-1}$ along the 'pursuit axis,' which was chosen for each experiment as the cardinal direction closest to orthogonal to the preferred direction of the MT neurons recorded at a given stimulation site. We measured learning in the 'learning axis,' which was defined as the cardinal axis closest to the preferred direction of the multiunit activity at the stimulation site and was always orthogonal to the pursuit axis. An example of a microstimulation-learning experiment is illustrated in Figure 1 for a site where the MT neurons preferred upwards motion. Each experiment began with a 'baseline block' of pre-learning 'probe trials' that consisted only of target motion in the pursuit direction (here rightward) to verify that before learning, rightward target motion elicited very little vertical eye motion (Fig. 1b, top). Next we presented 'learning trials' in which microstimulation was applied in MT for $300 \mathrm{~ms}$ starting $200 \mathrm{~ms}$ after the onset of target motion (Fig. 1a, middle). Stimulation at this site during ongoing pursuit elicited a small smooth eye movement with a major upward component ${ }^{15}$ (Fig. 1b, middle). After at least 100 learning trials, we assessed learning by measuring the vertical smooth eye movement on post-learning probe trials of rightward target motion in which stimulation was not delivered in MT (Fig. 1a, bottom); infrequent probe trials were interspersed among continuing learning trials.

The learned vertical eye movement had two components: an early peak followed by a later trough (Fig. 1b, bottom). The early component of learning was upward, towards the preferred direction of the neurons at the stimulation site, and peaked at around the time when microstimulation began in the learning trials. The later component of the learned eye movement was in the opposite direction, comprising a seemingly wrong-way response that was not observed when the instructive stimulus for learning was a change in target direction?

At each individual stimulation site, we customized the directions of target motion for the preferred directions of neurons at that site. For ease of presentation, however, we have rotated and reflected the data from each experiment, and will present our findings as if the learning direction represented by the site in MT were upward, and the pursuit direction used for real target motion were rightward. To quantify the amount of learning, we subtracted the vertical eye velocity recorded during probe trials in the baseline block from that recorded in identical probe trials toward the end of the learning block. Averaging the responses from all experiments in each monkey shows that the smooth eye movements evoked by stimulation on learning trials were unidirectional (Fig. 2a), whereas the learned eye movements were bidirectional (Fig. 2b). To represent variability across experiments, we also have summarized the learned eye velocities by plotting the amplitudes of the peak and trough for each individual experiment as a function of the times at which they occurred and connecting the points for each individual experiment with a line (Fig. 2c). The amplitudes of the early peaks showed a wide range, from very small to as large as $1^{\circ} \mathrm{s}^{-1}$, but the relationship between the time and amplitudes of the 
peaks was quite consistent: all lines in Figure 2c had similar, negative slopes. On average, the peaks in learned eye velocity occurred around the time corresponding to the onset of microstimulation on learning trials, whereas the oppositely-directed troughs occurred later, within the $300 \mathrm{~ms}$ interval that would have contained microstimulation on learning trials.

We found considerable variability across stimulation sites in the amplitudes of the learning induced by MT microstimulation (Fig. 2c). The variability did not correlate with any of our measures of the receptive field properties of each site, including direction tuning, preferred speed, spatial receptive field location and size, amplitude of the stimulation-evoked eye movement or whether the site responded better to the pursuit target or to the ensuing image motion that resulted from the motion of the eye across a stationary background (Supplementary Fig. 1).

\section{Origin of the oppositely directed component of learning}

The relative timing and directions of the two components of learning suggested that the first component was instructed by image motion signals resulting directly from MT stimulation, whereas the later, oppositely directed component might be instructed by real image motion signals. Microstimulation in MT evoked an eye movement on learning trials that drove the eye away from the target, causing target motion relative to the eye. Thus, stimulation provided consistent real image motion signals opposite to the direction of the stimulation-evoked eye movement. The real, target-related image motion signals would be delayed relative to those mimicked by stimulation in MT, because of (i) the latency between stimulation and the evoked eye movement and (ii) visual system processing delays. Thus, visual motion signals resulting from MT microstimulation and the ensuing target motion relative to the eye would predict oppositely directed, temporally segregated components of learning like those we observed (Figs. 1 and 2).

To test the hypothesis that target-related image motion was responsible for the second, oppositely directed component of learning we observed (Figs. 1 and 2), we stabilized the target on the eye and eliminated image motion during stimulation of MT by using the recorded eye position to drive target motion in real time. Microstimulation with target stabilization on learning trials evoked unidirectional eye movements (Fig. 3a) that were similar to those evoked without stabilization (Fig. 2a). However, the learned eye velocity expressed in probe trials was now also unidirectional and was always towards the preferred direction of the stimulation site (Fig. 3b). Individual experiments and trials also showed the basic features of the averages (Supplementary Fig. 2). Thus, target stabilization eliminated the second, oppositely directed component of learning. We conclude that visual signals resulting from microstimulation in MT and from real target motion relative to the eye are both sufficient to instruct directional learning in pursuit. Putting these signals in conflict causes a response that reflects the influence of both signals and their relative timing.

\section{Microstimulation-induced versus visually induced learning}

If MT provides instructive signals for pursuit learning under natural conditions, then we should find excellent quantitative agreement between the learned eye movements induced by microstimulation in MT and those induced by natural visual stimuli. Our previous experiments using a change in target direction to induce learning 7 provided only unidirectional instructive signals. Therefore, we conducted behavioral experiments with a natural visual stimulus designed to mimic the sequence of signals in MT and real image motion provided during microstimulation; we replaced MT stimulation with the motion of a visual background.

As before, each trial began when the monkey fixated a pursuit target; shortly thereafter a texture of stationary random dots appeared (Fig. 4, left). On pre-learning and post-learning probe trials, 
the visual background remained stationary as the pursuit target underwent ramp motion. On learning trials, the visual background remained stationary for the first $200 \mathrm{~ms}$ of pursuit target motion and then moved at $20^{\circ} \mathrm{s}^{-1}$ for $300 \mathrm{~ms}$ in a direction orthogonal to the direction of ongoing pursuit. In learning trials, the motion of the background evoked smooth eye movement towards the direction of background motion ${ }^{18}$ (Fig. $4 \mathrm{a}$ ). In subsequent probe trials, after at least 100 learning trials, the learned eye velocity had two components, one towards the direction of the background motion and one in the opposite direction (Fig. 4b). When the target was stabilized relative to the eye, the response to background motion on learning trials with stabilization (Fig. 4c) was similar to that without target stabilization, but the learned eye movement was entirely in the direction of the background motion (Fig. 4d). The second, oppositely directed component of the learned eye velocity again vanished. We conclude that both pursuit target-related and background motion are capable of instructing pursuit learning and that microstimulation in MT is an effective mimic of these signals.

Comparison of the timing of the learned eye velocities in the present study shows temporal differences between the learning instructed by MT microstimulation versus motion of a visual background. We plotted the magnitudes of the peak and trough of learned eye velocity as a function of their times for both microstimulation and background experiments (Fig. 5), aligned at the onsets of MT stimulation or background motion $(t=0)$. The general shape of the learned eye movements was similar, but those instructed by MT stimulation occurred earlier relative to the onset of the experimental manipulation than did those instructed by motion of the visual background. How can we understand these temporal differences?

If activity in MT were the instructive signal for both microstimulation and background-motion experiments, then the timing of the learned eye movements for the two learning conditions should be offset by an interval corresponding to the latency of MT neuronal responses to visual stimuli. To evaluate this prediction, we varied the interval between the onset of pursuit target motion and instructive background motion on different days. Aligning the learned eye velocities on the onset of pursuit target motion (Fig. 6a) reveals that changing the temporal intervals affected both the shape and timing of the learned eye velocity, as predicted from ref. 7 . Lengthening the interval between the onset of horizontal target motion and the vertical instructive signal from $100 \mathrm{~ms}$ to $150 \mathrm{~ms}$ or to $200 \mathrm{~ms}$ caused a longer latency and a larger peak in the learned eye movement towards the preferred direction of the stimulation site. There was a similar effect (Fig. 2b) when the interval between the onset of target motion and MT stimulation was varied in Monkey E.

We obtained the same temporal profile of learned eye movements only when we compared the learning induced by electrical stimulation for one interval with that induced by background motion that started 50 to $75 \mathrm{~ms}$ closer to the onset of visual target motion (Fig. 6b). In Monkey Q, the learned eye velocity for MT stimulation with an interval of $200 \mathrm{~ms}$ had a temporal structure that fell between that for the learned eye velocities from background motion with intervals of $100 \mathrm{~ms}$ and $150 \mathrm{~ms}$. In Monkey E, the best agreement in temporal structure occurred when the interval between the onset of target motion and instructive MT stimulation was more than $50 \mathrm{~ms}$ longer than the interval between target motion onset and instructive background motion. These data would be expected if MT neurons were activated by visual motion with a 50 to 75 ms latency ${ }^{19-22}$ and activity in MT provided an instructive signal for the learning induced by background motion as well as by microstimulation.

\section{DISCUSSION}

\section{Sensory instructive signals for pursuit learning}

Sensory error signals have been hypothesized to act as instructive signals for a number of forms of learning, and yet there are relatively few examples of vertebrate behaviors for which 
activation of a specific class of sensory neurons has been demonstrated to be sufficient to instruct learned changes in behavior; examples include classical conditioning of the eyelid response $^{23}$, auditory frequency discrimination 24 and now smooth pursuit eye movements.

We have shown that electrical microstimulation in area MT is sufficient as an instructive signal for learning in smooth pursuit eye movements. Further, there is excellent agreement in the expression of learning following instruction by MT microstimulation and by a natural visual stimulus. In particular, the learned eye movement instructed by MT stimulation was almost identical to that instructed by motion of a visual background when the comparison took account of the latency of MT responses to visual motion. Relative to the onset of pursuit target motion in our learning paradigm, MT stimulation had to occur 50 to $75 \mathrm{~ms}$ later than background motion, exactly as expected if visual signals took 50 to $75 \mathrm{~ms}$ longer to reach the sites of instruction than those produced by activating MT directly. The similarity of the learning in the two situations implies that activity in MT represents the instructional signals for learning even when the instructional stimulus is provided by real image motion. Although other brain regions have similar response latencies and also could contribute instructional signals for pursuit learning, our findings establish a clear role for MT.

For instructional signals that arise from both MT microstimulation and real image motion, the temporal relation between the instructive stimulus and the learned eye velocity was notable. We have previously shown ${ }^{7}$ that learned eye movements are timed to correspond to the time of a change in direction of the pursuit target. In the present study, we found that the learned eye velocity followed the sequence of two image motion instructive signals: first in one direction and then in the opposite direction owing to target motion with respect to the eye. We were able to link the second component of learning to the oppositely directed image motion by experiments in which the target was stabilized on the eye in the learning trials, to eliminate oppositely directed image motion.

The fact that the pursuit system learned to make a rapid sequence of eye motion in one direction and then the other instead of averaging the two opposing instructional signals over time indicates that it is specialized for learning temporal properties of the instructional signal on a time scale of $100 \mathrm{~ms}$. Further, both background motion and microstimulation were effective at instructing pursuit learning even if they originated from parts of the visual field that were clearly separate from the foveal location of the pursuit target. Thus, learning in the pursuit system seems to operate more like a machine than like a cognitive process ${ }^{25}$. Pursuit learning responds to the association of ongoing pursuit and specific temporal sequences of image motion and is driven by spatially non-specific instructional signals.

Because MT microstimulation caused an eye movement in the learning trials, we cannot rule out the possibility that the visual signals from MT have been converted at least partially into motor coordinates before reaching the site of learning. In future experiments, subthreshold stimulation of MT might help resolve this issue ${ }^{26}$. For now, several aspects of our data imply that the instructional signals for pursuit learning are not related directly to eye movements. First, in experiments that did not use target stabilization, the eye movement responses on learning trials were unidirectional, but the learned eye movements had a component in the opposite direction. Second, target stabilization dissociated these two eye movements: it had only minimal effects on the eye movement on learning trials, but it profoundly altered the shape of the learned eye movement. Finally, in microstimulation and behavioral experiments, the first peak of the learned eye velocity is timed to coincide with the time of MT activation and not with the time of the evoked eye movement. 


\section{Implications for sites and mechanisms of learning}

Our experiments do not speak directly to the site of learning for pursuit other than to indicate that the site of learning must receive major inputs from MT. A previous study 27 provided evidence that the site of pursuit learning is downstream from the frontal pursuit area. Our finding that the site of pursuit learning is also downstream from area MT pushes candidate loci into subcortical structures, including brainstem precerebellar nuclei and the cerebellum itself $^{28}$. In primates, MT is a major source of visual motion signals to the cerebellum, accessing both cerebellar mossy fibers and climbing fibers via the dorsolateral pontine nucleus and the nucleus of the optic tract ${ }^{29-31}$.

One aspect of our data that provides insight into potential neural mechanisms for pursuit learning is that the pursuit system compensates for the temporal delay in the instructive signal from MT. Learned eye movements began, and in some experiments reached their peaks, before the time when stimulation was delivered on learning trials. This finding provides a constraint for potential plasticity mechanisms that might mediate pursuit learning. Specifically, these plasticity mechanisms would be expected to have asymmetrical temporal requirements, as has been proposed for some cerebellar-based learned behaviors 32,33 and demonstrated for several forms of cerebellar plasticity $34-37$.

In conclusion, we have demonstrated that visual signals arising in visual area MT are a powerful instructive signal for pursuit learning, cementing MT in the pathway for signals that instruct learning. We are struck that the organization of the pursuit circuit bears strong resemblance to the organization of circuits for a wide range of motor behaviors. All use inputs from sensory cortex, cortico-cortical pathways through the parietal sensory-motor areas and recurrent loops through both the cerebellum and the basal ganglia ${ }^{38,39}$. Many researchers have proposed that the cerebellum may be responsible for error-correcting learning for a wide range of behaviors ${ }^{40-42}$, and pursuit has become an important model system for understanding errorcorrecting learning. We propose that the nature of the instructive signals and the sites and mechanisms of learning in pursuit may provide a framework that can be generalized to a wide range of learned motor behaviors.

\section{METHODS}

Animals

All experimental procedures were approved in advance by the Institutional Animal Care and Use Committee of the University of California, San Francisco and conformed to federal guidelines. Two male rhesus monkeys (Macaca mulatta, 7-12 kg) served as subjects, and their eye movements were recorded with the magnetic search coil method. Surgical and training procedures to prepare monkeys for the study have been described previously 43,44 . Both monkeys were well trained on pursuit tasks but naive to pursuit learning experiments. Experiments were conducted approximately five times per week and lasted 2-5 hours. All experiments were conducted in a nearly dark room.

\section{Visual stimuli}

Visual stimuli were presented on an analog oscilloscope (Hewlett Packard 1304A) with a refresh rate of $250 \mathrm{~Hz}$. The display was positioned $30 \mathrm{~cm}$ from the monkey and subtended $41^{\circ}$ horizontal $\times 34^{\circ}$ vertical of visual angle. Target position and velocity were specified through the user interface on a DEC Alpha UNIX workstation and were controlled by the outputs of two 16-bit digital-to-analog converters on a digital signal processing board in a PC.

A small bright spot was presented as a pursuit target in individual trials. Each trial began with a fixation interval of a duration that was randomized between 500 and 1,000 ms. The target 
then underwent 'step-ramp' motion to minimize the occurrence of catch-up saccades. The target stepped away from the fixation point by $2.5^{\circ}$ (Monkey Q) or 3.0 (Monkey E) and simultaneously began moving at $20^{\circ} \mathrm{s}^{-1}$ towards the fixation point. Monkeys were rewarded with a drop of fluid if they kept eye position close to target position throughout the trial (see below). They completed approximately 1,500 pursuit trials, plus several hundred receptive field-mapping trials, in each daily experiment.

In some experiments, we also presented a visual background that consisted of randomly placed dots at an average density of $0.09 \mathrm{dots} / \mathrm{deg}^{2}$. The dots were one-sixteenth as bright as the pursuit target. To avoid spatial overlap of the target and the background, dots were visible within two separate invisible apertures of $38^{\circ} \times 14^{\circ}$, one above and one below the horizontally-moving pursuit target. Thus, the pursuit target was centered vertically on a $6^{\circ}$-tall horizontal stripe in which background dots never appeared. The stripe between the two apertures was only slightly taller than the average separation between dots, thereby minimizing the interruption of the background pattern while preventing the background from introducing ambiguity as to the task requirements by overlapping the path traversed by the target. The placement of individual dots within the background pattern was varied randomly on a trial-by-trial basis. Motion of the visual background consisted of $100 \%$ coherent vertical motion of the dots at $20^{\circ} \mathrm{s}^{-1}$ within the stationary, virtual apertures.

In a subset of our experiments we stabilized targets with respect to the moving eye ${ }^{45}$ to eliminate image motion resulting from eye motion relative to the target on learning trials. The position of the eye was sampled every millisecond and fed back to the computer so that it could be used to drive target motion in real time. We used the monkeys' eye movements in response to small position and velocity errors to verify the accuracy of the calibration of the eye coil $^{45}$ and ensure accurate image stabilization. Comparison of the eye movements evoked by target motion with and without target stabilization showed neither the saccades nor the smooth eye accelerations that would have been predicted by systematic errors in stabilization. In a subset of our experiments we selectively stabilized the target only along the (horizontal or vertical) learning axis and not along the orthogonal pursuit axis. Our results did not depend on whether the target was stabilized along both or just one of the axes.

\section{MT recording and microstimulation}

We recorded in and stimulated MT in three hemispheres of two monkeys (left and right hemispheres of Monkey Q, left only in Monkey E). We used tungsten microelectrodes with impedances from $800 \mathrm{~K} \Omega$ to $1.2 \mathrm{M} \Omega$ measured at $1 \mathrm{kHz}$ (Frederick Haer). Area MT was identified as described previously 44 based on stereotaxic coordinates, directional response properties of MT neurons, receptive field sizes, retinotopic organization, and activity recorded in surrounding cortical areas. We used a vertical approach to MT, so that we usually were able to identify the middle superior temporal area (MST) and the lumen of the superior temporal sulcus before the electrode entered MT. Once we understood the topographical organization of MT in each hemisphere, we attempted to find sites with receptive field locations in the central $10^{\circ}$ of the visual field.

Once we entered MT, we searched for a site where the multiunit activity indicated that nearby neurons shared a common direction preference for the motion (usually at $16^{\circ} \mathrm{s}^{-1}$ ) of dots that were placed randomly within a $38^{\circ} \times 30^{\circ}$ aperture in the visual hemifield contralateral to the recording site. Only strongly direction-selective sites with reasonably Gaussian direction tuning curves were selected for further experimentation. Once a potential stimulation site had been identified we characterized its direction and speed preferences for motion of patches of dots, as well as its spatial receptive field location and size, its responses to motion of pursuit targets and background textures, and the effects of microstimulation on eye movements during ongoing pursuit. 
We delivered biphasic current pulses under the control of a Grass S88 stimulator. Pulses had intensities of 30-50 $\mu \mathrm{A}$ and durations of $0.2 \mathrm{~ms}$, and occurred at a rate of $200 \mathrm{~Hz}$ in trains of 300 -ms duration. In agreement with the previous finding 46 that small changes in electrode position away from the center of a direction column can reduce the effectiveness of microstimulation in MT, we found that microstimulation at sites with weak or ambiguous direction tuning profiles usually failed to elicit smooth eye movements even during ongoing pursuit. However, stimulation nearly always evoked a directional eye movement at the relatively low currents we used, if performed at sites with strong multiunit responses to motion and clear direction tuning. In contrast to previous studies in which stimulation coincided with the onset of pursuit ${ }^{15,47}$, the stimulation-evoked eye movements we observed during pursuit maintenance were consistently towards (and not away from) the preferred direction of the stimulation site. Because we required the motion signal injected through stimulation to be as directional as possible, we abandoned sites at which microstimulation applied during ongoing pursuit failed to produce a smooth eye movement. The presence of a stimulation-evoked eye movement also allowed us to monitor the continued effectiveness of the stimulation site during long experiments.

\section{Behavioral control}

The learning experiments were similar in design to previous studies on pursuit learning 6,7 , 27 and consisted of two blocks of trials: a baseline block and a learning block. Each block consisted of one or more trial types presented in varying ratios in pseudorandom order. The baseline block consisted of probe trials in which the target moved along the pursuit axis without any additional experimental manipulations. Before proceeding to a learning block of trials, we confirmed the consistency and stability of learning axis eye velocities on baseline probe trials. If the eye velocities were not stable across different fractions of the baseline block, we terminated the experiment for that day to avoid contamination of our data from fluctuations caused by poor pursuit performance. In up to $10 \%$ of the trials in the baseline block, microstimulation was applied in MT starting 200 or $250 \mathrm{~ms}$ after the onset of target motion. The learning block contained at least $80 \%$ learning trials and $20 \%$ or fewer probe trials. Learning trials always consisted of pursuit target motion identical to that of probe trials. In addition, either microstimulation in MT or motion of a visual background was delivered for $300 \mathrm{~ms}$ beginning at a fixed interval (100-250 ms, usually $200 \mathrm{~ms})$ after the onset of target motion. In some experiments, the target was stabilized on learning trials during the $300 \mathrm{~ms}$ segment when microstimulation or visual background motion was delivered. Probe trials in the learning block were identical to probe trials in the baseline block.

Monkeys were generally required to keep their eyes within a $3^{\circ}$ window around the pursuit target to complete a trial and receive a fluid reward. Our experimental manipulations caused deviations in eye position of less than $1^{\circ}$ and therefore should not have affected the monkey's likelihood of successfully completing the trial. However, to be absolutely certain that microstimulation, background motion or learning did not affect reward probabilities, we opened the fixation window along the learning axis to $5^{\circ}$ during the segment of the learning trials in which microstimulation was applied or the visual background moved and during the corresponding segments on probe trials. In an attempt to eliminate potential confusion between the target and the dots in the visual background experiment, we also conducted the background experiments with the fixation window in only the learning axis narrowed to as little as $1^{\circ}$. Learning was unaffected by these changes in fixation requirements; we obtained excellent task performance and clear, bidirectional learning with the fixation window either widened or narrowed along the learning axis. 


\section{Data analysis}

Details of our data acquisition have been published before ${ }^{7,44}$. Data from any given trial were used for analysis only if the trial had been completed successfully by the monkey. Saccades were marked by hand using an interactive computer program, and the portions of the eye velocity traces corresponding to saccades were treated as missing data. If a saccade occurred during the segment corresponding to microstimulation or visual background motion, then the entire trial was excluded from analysis. Due to the small size of the learned eye movements, we aligned responses to identical stimuli on the onset of target motion or the time of microstimulation or visual background motion and computed time averages of eye velocity. Responses to MT stimulation were isolated by subtracting the eye velocity along the learning axis on trials in which microstimulation was not delivered from that when stimulation occurred. Learning was assessed by computing the difference between averaged eye velocity in the learning axis for probe trials near the end of the learning block and those in the baseline block. No attempt was made to assess the amount of learning until $\sim 100$ learning trials had been completed.

\section{ACKNOWLEDGMENTS}

We thank M. Brainard, D. Copenhagen, M. Mauk, W. Newsome, M. Orger, R. Ramachandran, P. Sabes and D. Bodznick for helpful discussion; L. Bocskai, D. Kleinhesselink, K. McGary, S. Ruffner and D. Wolfgang-Kimball for excellent technical support and S. Tokiyama, E. Montgomery, B. St. Amant and K. MacLeod for superb animal care. This work was supported by the Howard Hughes Medical Institute, US National Institutes of Health grant NS34835, and an Achievement Rewards for College Scientists fellowship to M.R.C.

\section{References}

1. Schultz W, Dickinson A. Neuronal coding of prediction errors. Annu. Rev. Neurosci 2000;23:473500. [PubMed: 10845072]

2. Rescorla, RA.; Wagner, AR. A theory of pavlovian conditioning: variations in the effectiveness of reinforcement and nonreinforcement.. In: Black, AH.; Prokasy, WF., editors. Classical Conditioning II: Current Research and Theory. Appleton Century Crofts; New York: 1972. p. 64-99.

3. Ito M. Neural design of the cerebellar motor control system. Brain Res 1972;40:81-84. [PubMed: 4338265]

4. Rashbass C. The relationship between saccadic and smooth tracking eye movements. J. Physiol. (Lond.) 1961;159:326-338. [PubMed: 14490422]

5. Robinson DA. The mechanics of human smooth pursuit eye movement. J. Physiol. (Lond.) 1965;180:569-591. [PubMed: 5846794]

6. Kahlon M, Lisberger SG. Coordinate system for learning in the smooth pursuit eye movements of monkeys. J. Neurosci 1996;16:7270-7283. [PubMed: 8929434]

7. Medina JF, Carey MR, Lisberger SG. The representation of time for motor learning. Neuron 2005;45:157-167. [PubMed: 15629710]

8. Boman DK, Hotson JR. Predictive smooth pursuit eye movements near abrupt changes in motion direction. Vision Res 1992;32:675-689. [PubMed: 1413552]

9. Maunsell JH, Van Essen DC. Functional properties of neurons in middle temporal visual area of the macaque monkey. I. Selectivity for stimulus direction, speed, and orientation. J. Neurophysiol 1983;49:1127-1147. [PubMed: 6864242]

10. Albright TD. Direction and orientation selectivity of neurons in visual area MT of the macaque. J. Neurophysiol 1984;52:1106-1130. [PubMed: 6520628]

11. Newsome WT, Wurtz RH, Dursteler MR, Mikami A. Deficits in visual motion processing following ibotenic acid lesions of the middle temporal visual area of the macaque monkey. J. Neurosci 1985;5:825-840. [PubMed: 3973698]

12. Newsome WT, Wurtz RH, Komatsu H. Relation of cortical areas MT and MST to pursuit eye movements II. Differentiation of retinal from extraretinal inputs. J. Neurophysiol 1988;60:604-620. [PubMed: 3171644] 
13. Lisberger SG, Movshon JA. Visual motion analysis for pursuit eye movements in area MT of macaque monkeys. J. Neurosci 1999;19:2224-2246. [PubMed: 10066275]

14. Komatsu H, Wurtz RH. Modulation of pursuit eye movements by stimulation of cortical areas MT and MST. J. Neurophysiol 1989;62:31-47. [PubMed: 2754480]

15. Groh JM, Born RT, Newsome WT. How is a sensory map read out? Effects of microstimulation in visual area MT on saccades and smooth pursuit eye movements. J. Neurosci 1997;17:4312-4330. [PubMed: 9151748]

16. Salzman CD, Murasugi CM, Britten KH, Newsome WT. Microstimulation in visual area MT: effects on direction discrimination performance. J. Neurosci 1992;12:2331-2355. [PubMed: 1607944]

17. Bisley JW, Zaksas D, Pasternak T. Microstimulation of cortical area MT affects performance on a visual working memory task. J. Neurophysiol 2001;85:187-196. [PubMed: 11152719]

18. Kodaka Y, Miura K, Suehiro K, Takemura A, Kawano K. Ocular tracking of moving targets: effects of perturbing the background. J. Neurophysiol 2004;91:2474-2483. [PubMed: 14762158]

19. Schmolesky MT, et al. Signal timing across the macaque visual system. J. Neurophysiol 1998;79:3272-3278. [PubMed: 9636126]

20. Raiguel SE, Xiao DK, Marcar VL, Orban GA. Response latency of macaque area MT/V5 neurons and its relationship to stimulus parameters. J. Neurophysiol 1999;82:1944-1956. [PubMed: 10515984]

21. Cook EP, Maunsell JH. Dynamics of neuronal responses in macaque MT and VIP during motion detection. Nat. Neurosci 2002;5:985-994. [PubMed: 12244324]

22. Bair W, Cavanaugh JR, Smith MA, Movshon JA. The timing of response onset and offset in macaque visual neurons. J. Neurosci 2002;22:3189-3205. [PubMed: 11943820]

23. Mauk MD, Steinmetz JE, Thompson RF. Classical conditioning using stimulation of the inferior olive as the unconditioned stimulus. Proc. Natl. Acad. Sci. USA 1986;83:5349-5353. [PubMed: 3460097]

24. Talwar SK, Gerstein GL. Reorganization in awake rat auditory cortex by local microstimulation and its effect on frequency-discrimination behavior. J. Neurophysiol 2001;86:1555-1572. [PubMed: 11600620]

25. Kowler E. Cognitive expectations, not habits, control anticipatory smooth oculomotor pursuit. Vision Res 1989;29:1049-1057. [PubMed: 2617852]

26. Moore T, Armstrong KM. Selective gating of visual signals by microstimulation of frontal cortex. Nature 2003;421:370-373. [PubMed: 12540901]

27. Chou IH, Lisberger SG. The role of the frontal pursuit area in learning in smooth pursuit eye movements. J. Neurosci 2004;24:4124-4133. [PubMed: 15115807]

28. Kahlon M, Lisberger SG. Changes in the responses of Purkinje cells in the floccular complex of monkeys after motor learning in smooth pursuit eye movements. J. Neurophysiol 2000;84:29452960. [PubMed: 11110823]

29. Distler C, Mustari MJ, Hoffmann KP. Cortical projections to the nucleus of the optic tract and dorsal terminal nucleus and to the dorsolateral pontine nucleus in macaques: a dual retrograde tracing study. J. Comp. Neurol 2002;444:144-158. [PubMed: 11835187]

30. Alley K, Baker R, Simpson JI. Afferents to the vestibulo-cerebellum and the origin of the visual climbing fibers in the rabbit. Brain Res 1975;98:582-589. [PubMed: 1182539]

31. Brodal P. The pontocerebellar projection in the rhesus monkey: an experimental study with retrograde axonal transport of horseradish peroxidase. Neuroscience 1979;4:193-208. [PubMed: 106327]

32. Raymond JL, Lisberger SG. Neural learning rules for the vestibulo-ocular reflex. J. Neurosci 1998;18:9112-9129. [PubMed: 9787014]

33. Medina JF, Nores WL, Ohyama T, Mauk MD. Mechanisms of cerebellar learning suggested by eyelid conditioning. Curr. Opin. Neurobiol 2000;10:717-724. [PubMed: 11240280]

34. Bell CC, Han VZ, Sugawara Y, Grant K. Synaptic plasticity in a cerebellum-like structure depends on temporal order. Nature 1997;387:278-281. [PubMed: 9153391]

35. Wang SS, Denk W, Hausser M. Coincidence detection in single dendritic spines mediated by calcium release. Nat. Neurosci 2000;3:1266-1273. [PubMed: 11100147]

36. Chen C, Thompson RF. Temporal specificity of long-term depression in parallel fiber-Purkinje synapses in rat cerebellar slice. Learn. Mem 1995;2:185-198. [PubMed: 10467575] 
37. Brenowitz SD, Regehr WG. Associative short-term synaptic plasticity mediated by endocannabinoids. Neuron 2005;45:419-431. [PubMed: 15694328]

38. Middleton FA, Strick PL. Basal ganglia and cerebellar loops: motor and cognitive circuits. Brain Res. Brain Res. Rev 2000;31:236-250. [PubMed: 10719151]

39. Tian JR, Lynch JC. Subcortical input to the smooth and saccadic eye movement subregions of the frontal eye field in Cebus monkey. J. Neurosci 1997;17:9233-9247. [PubMed: 9364070]

40. Doya K. Complementary roles of basal ganglia and cerebellum in learning and motor control. Curr. Opin. Neurobiol 2000;10:732-739. [PubMed: 11240282]

41. Hikosaka O, Nakamura K, Sakai K, Nakahara H. Central mechanisms of motor skill learning. Curr. Opin. Neurobiol 2002;12:217-222. [PubMed: 12015240]

42. Jueptner M, Frith CD, Brooks DJ, Frackowiak RS, Passingham RE. Anatomy of motor learning. II. Subcortical structures and learning by trial and error. J. Neurophysiol 1997;77:1325-1337. [PubMed: 9084600]

43. Carey MR, Lisberger SG. Signals that modulate gain control for smooth pursuit eye movements in monkeys. J. Neurophysiol 2004;91:623-631. [PubMed: 14561686]

44. Churchland MM, Lisberger SG. Shifts in the population response in the middle temporal visual area parallel perceptual and motor illusions produced by apparent motion. J. Neurosci 2001;21:9387_ 9402. [PubMed: 11717372]

45. Morris EJ, Lisberger SG. Different responses to small visual errors during initiation and maintenance of smooth-pursuit eye movements in monkeys. J. Neurophysiol 1987;58:1351-1369. [PubMed: 3437336]

46. Murasugi CM, Salzman CD, Newsome WT. Microstimulation in visual area MT: effects of varying pulse amplitude and frequency. J. Neurosci 1993;13:1719-1729. [PubMed: 8463847]

47. Born RT, Groh JM, Zhao R, Lukasewycz SJ. Segregation of object and background motion in visual area MT: effects of microstimulation on eye movements. Neuron 2000;26:725-734. [PubMed: 10896167]

\section{Supplementary Material}

Refer to Web version on PubMed Central for supplementary material. 
a
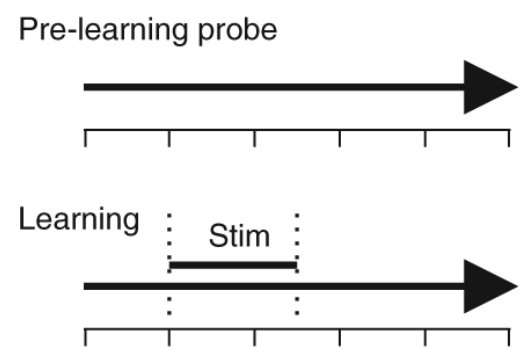

Post-learning probe

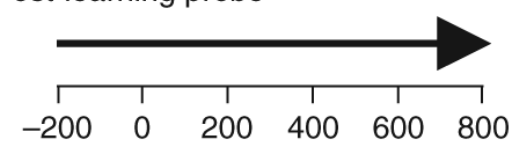

b
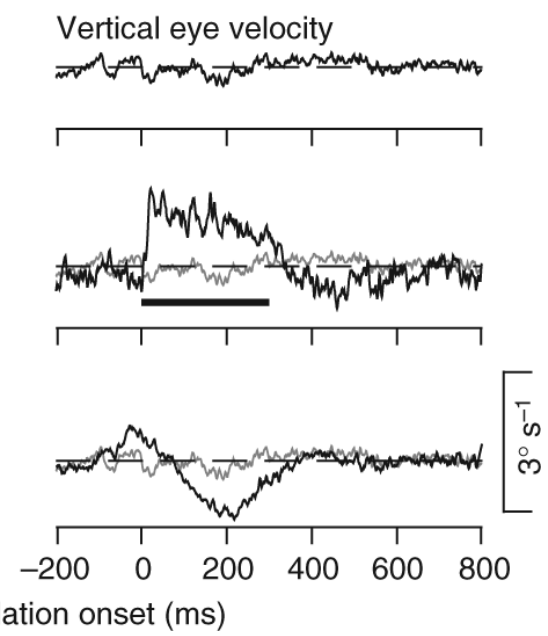

Figure 1.

A sample experiment using MT microstimulation to instruct learning. (a) Schematic of the experimental design for a learning experiment. From top to bottom, the three schematics show the probe trials in the baseline block and the learning and probe trials in the learning block. The bold arrow indicates the rightward motion of the pursuit target as a function of time. The vertical dotted lines and the horizontal bar labeled 'Stim' indicate the interval when MT was stimulated in the learning trials. (b) Averaged eye velocity along the learning axis, which was vertical in this experiment. In each panel, the black trace shows vertical eye velocity as a function of time and the horizontal dashed line indicates zero eye velocity. In the second and third panels, the gray trace reiterates the vertical eye velocity in the pre-learning probe trials, also shown by the black trace in the top panel. For this and all figures, learning trial data are from early in the learning block, before learning has occurred, and learned eye movements on probe trials are after at least 100 learning trials. Upward deflection of the velocity traces corresponds to upward eye motion. 

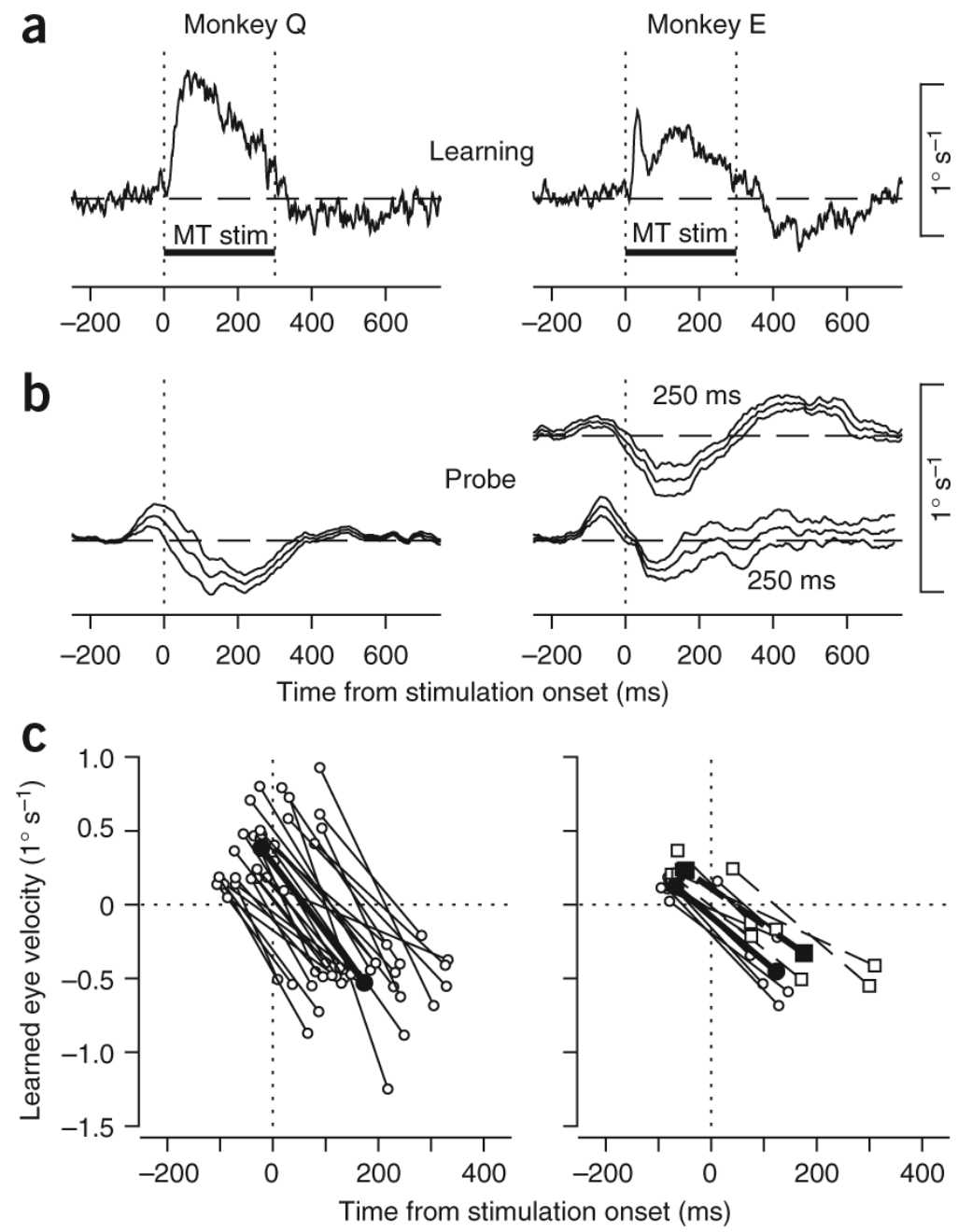

Figure 2.

Summary of learned eye velocity when stimulation in MT provided the instructive signal for learning. (a) Time course of averaged eye velocity responses to MT stimulation in learning trials. The two vertical dotted lines and the horizontal bar labeled 'MT stim' indicate the interval of microstimulation. Data were taken from the first few learning trials before measurable learning had occurred. (b) Time course of learned eye movements ( \pm s.e.m.) along the learning axis, measured in infrequent probe trials. Microstimulation started $200 \mathrm{~ms}$ after the onset of target motion in Monkey Q and either 200 or $250 \mathrm{~ms}$ after the onset of target motion for Monkey E. Data in $\mathbf{a}$ and $\mathbf{b}$ are averages across experimental days ( $n=30$ for Monkey $\mathbf{Q}, n=6$ for each temporal interval for Monkey E). (c) Summary of the two components of learned eye movements. Each point plots the positive or negative peak of learned eye velocity as a function of the time of the peak. Each pair of open symbols connected by a line shows data from an individual experiment. Filled symbols connected by bold lines show averages across all experiments. For Monkey E, circles and continuous lines show data obtained with an interval of $200 \mathrm{~ms}$; squares and dashed lines show data obtained with an interval of $250 \mathrm{~ms}$. Positive values of eye velocity indicate eye motion in the learning direction. 

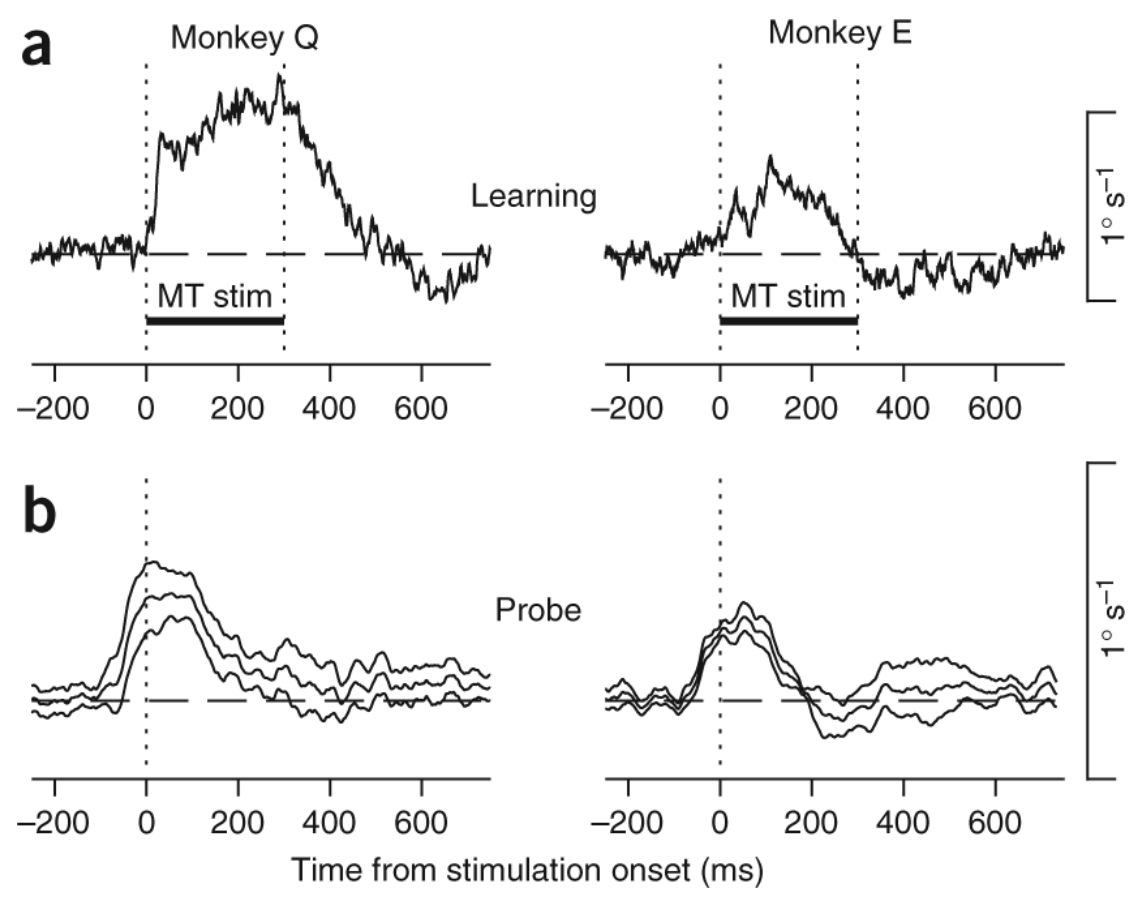

Figure 3.

Summary of learned eye velocity when stimulation in MT combined with target stabilization provided the instructive signal for learning. (a) Time course of averaged eye velocity responses to MT stimulation in learning trials. The two vertical dotted lines and the horizontal bar labeled 'MT stim' indicate the interval of microstimulation and target stabilization. Data were taken from the first few learning trials before measurable learning had occurred. (b) Time course of learned eye movements ( \pm s.e.m.) along the learning axis, measured in infrequent probe trials. Microstimulation started $200 \mathrm{~ms}$ after the onset of target motion in both monkeys. Data show averages across eight and five experimental days for Monkeys Q and E, respectively. 


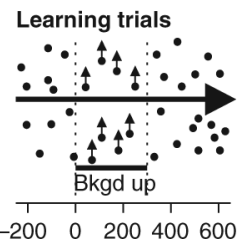

a
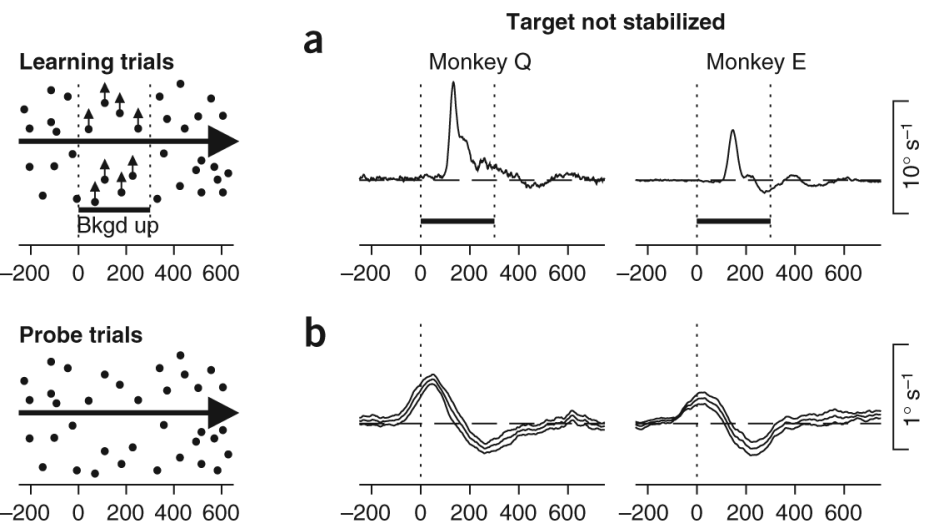

b

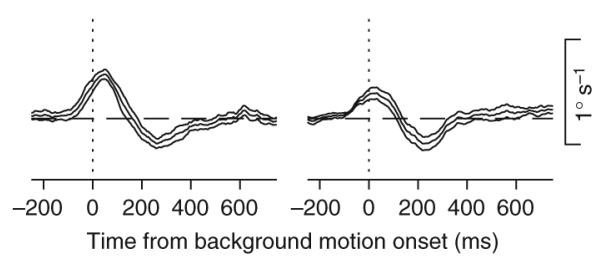

C

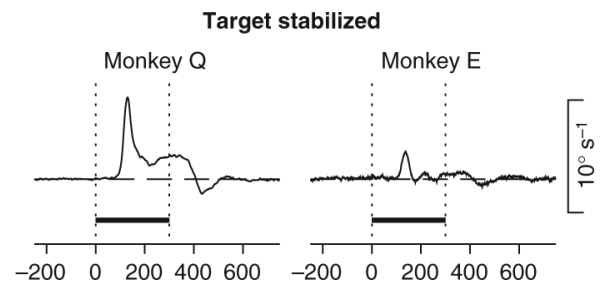

d

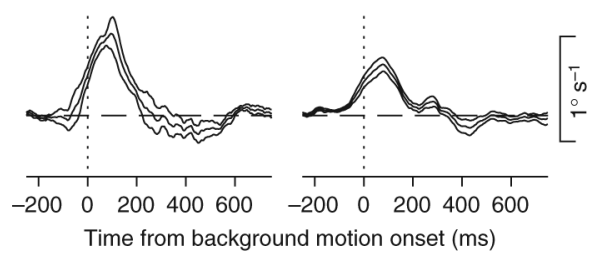

Figure 4.

Learning instructed by motion of a visual background during orthogonal target motion. The diagrams on the left summarize the visual stimuli used to induce learning (top) and to evaluate the learned eye velocity (bottom). The interval indicated by the two vertical dotted lines and the horizontal bar labeled 'Bkgd up' indicates the time when the background stimulus moved upwards, starting $200 \mathrm{~ms}$ after target motion onset. (a,b) Learning instructed by background motion without target stabilization. (c,d) Learning instructed by background motion with target stabilization on the learning trials. (a,c) Averaged eye velocity responses during the first few learning trials, before measurable learning had occurred. Data are averages across experimental days ( $n=7$ for each monkey) and are plotted as a function of time from onset of visual background motion (time 0). (b,d) Learned eye movements on probe trials after at least 100 learning trials. Data are plotted as mean \pm s.e.m. as a function of the time that visual background motion began on learning trials. 


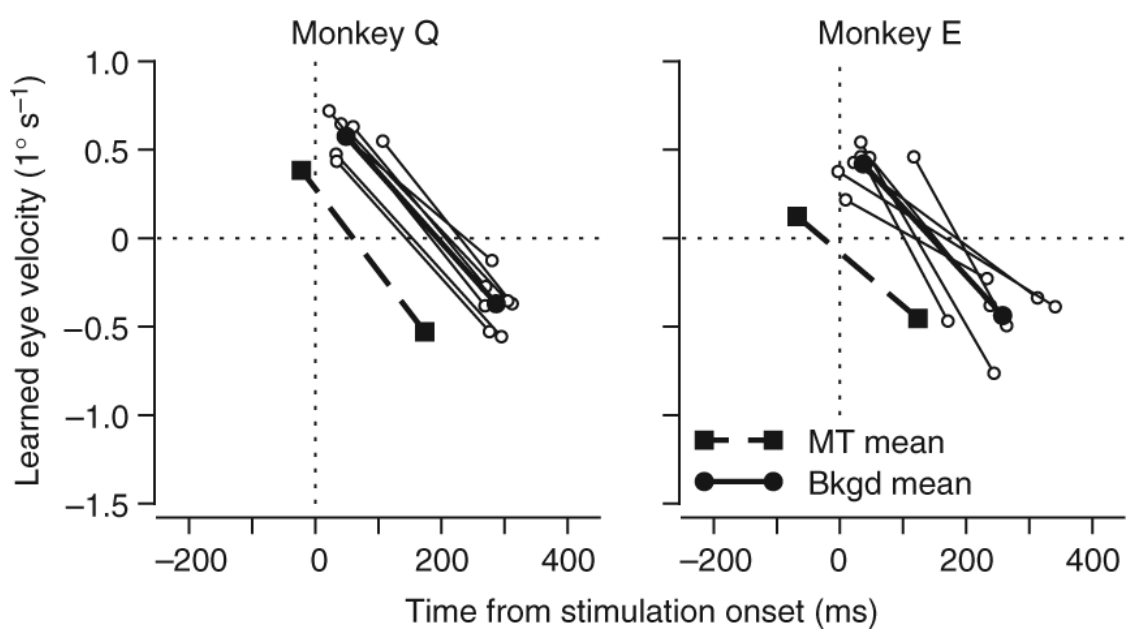

Figure 5.

Temporal relationship between learning instructed by MT microstimulation and visual background motion. Each point plots the positive or negative peak of learned eye velocity as a function of the time of the peak. Each pair of open symbols connected by a line shows data from an individual visual background motion experiment. Filled circles connected by bold lines show averages across all background experiments. Filled squares and bold dashed lines show averages of the learning instructed by MT stimulation. Positive values of eye velocity indicate eye motion in the learning direction. Time zero indicates the onset of visual background motion or MT microstimulation in learning trials, which was $200 \mathrm{~ms}$ after target motion onset in both cases. 

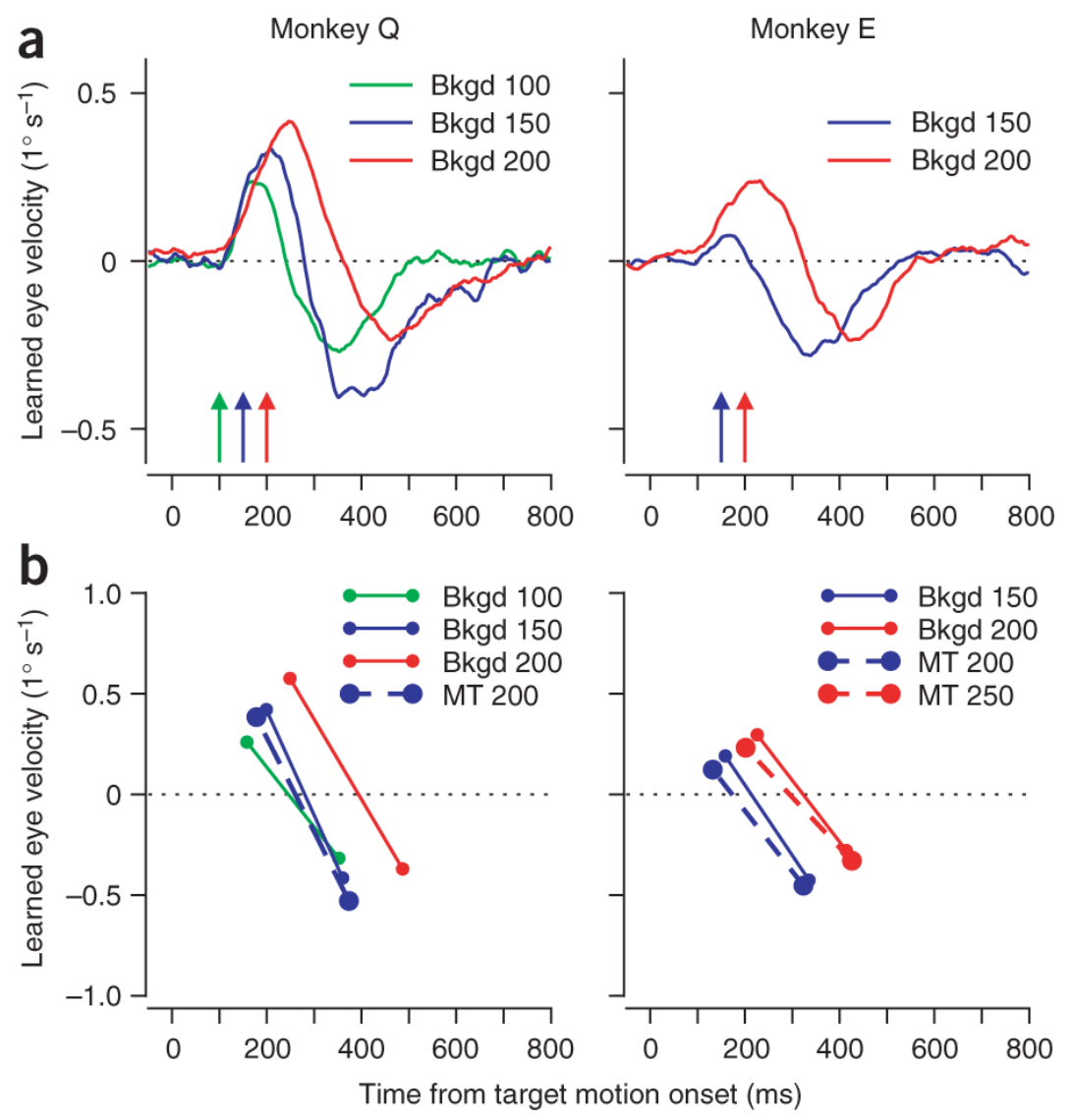

Figure 6.

Comparison of learning instructed by MT stimulation and visual background motion at different times after the onset of pursuit target motion. (a) Average time courses of learned eye velocity: green, blue and red traces show results for experiments in which learning was induced by visual background motion that began 100,150 or $200 \mathrm{~ms}$ after the onset of pursuit target motion, respectively. The colored arrows indicate the times of visual background motion in the learning trials for each experiment. (b) Each point plots the average positive or negative peak of the learned eye velocity as a function of the time of the peak. Each pair of symbols connected by a line shows data from an individual learning condition. Bold circles connected by dashed lines indicate the results of learning instructed by stimulation of MT. Smaller circles connected by thin lines indicate the results of learning instructed by background motion. For experiments with background motion, the colors have the same meaning as in a. For experiments with MT stimulation, the color scheme has been shifted by $50 \mathrm{~ms}$ : blue and red symbols indicate the MT stimulation occurred 200 or $250 \mathrm{~ms}$ after the onset of pursuit target motion in learning trials. In both $\mathbf{a}$ and $\mathbf{b}$, time zero indicates the onset of pursuit target motion. 